\title{
ADJUNCTION SPACES AND THE HEREDITARY PROPERTY
}

\author{
BYRON H. MCCANDLESS ${ }^{1}$
}

Let $X$ and $Y$ be spaces, $A$ a closed subset of $X$, and $f: A \rightarrow Y$ a map (i.e., a continuous transformation). Let $Z$ be the adjunction space obtained by adjoining $X$ to $Y$ by means of $f[5$, p. 121]. There are two natural maps, $k: X \rightarrow Z$ and $j: Y \rightarrow Z$, and a set $U$ in $Z$ is open if and only if $k^{-1}(U)$ is open in $X$ and $j^{-1}(U)$ is open in $Y$. The map $j$ is an imbedding, and it is easily seen that $j(Y)$ is a closed subset of $Z$. By identifying $Y$ with its homeomorphic image $j(Y)$ we may consider $Y$ to be a closed subset of $Z$. With this understanding, a set $U$ in $Z$ is open if and only if $k^{-1}(U)$ is open in $X$ and $U \cap Y$ is open in $Y$. Then it can be shown that $k$ is an extension of $f$ over $X$ (with respect to $Z$ ) and that $k \mid X-A$ maps $X-A$ homeomorphically onto $Z-Y$.

A topological property $P$ of spaces is said to be preserved by the adjunction space $Z$ if and only if $Z$ has $P$ whenever $X$ and $Y$ both have $P$. In this paper we are interested in certain hereditary properties that are preserved by the adjunction space. Iséki has shown $[7$, p. 144] that the adjunction space preserves hereditary normality (or complete normality, as it is usually called). Iséki's proof makes use of separated subsets of $Z$, and holds only in the case of complete normality. We will see that Iséki's theorem follows as a special case of one of our theorems; in fact we shall prove that the adjunction space preserves any property "Hereditary $P$ " such that the property $P$ satisfies

(a) $Z$ preserves $P$;

(b) If every open subset of a space $X$ has $P$, then every subset of $X$ has $P$.

The idea of the proof is to notice that, with a trivial exception, every open subset of an adjunction space $Z$ can be considered, in a natural way, as an adjunction space.

Let $Z^{\prime}$ be a subspace of the adjunction space $Z$ and define $X^{\prime}=k^{-1}\left(Z^{\prime}\right), \quad Y^{\prime}=Y \cap Z^{\prime}, A^{\prime}=A \cap X^{\prime}$, and $f^{\prime}=f \mid A^{\prime}$. Then $A^{\prime}$ is a closed subset of $X^{\prime}$ and $f^{\prime}$ is a map of $A^{\prime}$ into $Y^{\prime}$. We will show that if $Z^{\prime}$ is an open subspace of $Z$, then $Z^{\prime}$ is (with the exception noted) the adjunction space obtained by adjoining $X^{\prime}$ to $Y^{\prime}$ by means of $f^{\prime}$. The

Presented to the Society, June 18, 1966; received by the editors July 29, 1966.

1 Research supported by the Bureau for Faculty Research at Western Washington State College, summer, 1966. 
same is true if $Z^{\prime}$ is a closed subspace of $Z$, although the latter statement does not seem to be especially significant.

Lemma 1. If $Z^{\prime}$ is an open subspace of $Z$ and if $A^{\prime} \neq \varnothing$, then $Z^{\prime}$ is the adjunction space obtained by adjoining $X^{\prime}$ to $Y^{\prime}$ by means of $f^{\prime}$.

Proof. We are concerned with two topologies for $Z^{\prime}$ which are defined in different ways: the subspace topology inherited from $Z$, and the adjunction space topology. We shall show that these two topologies coincide.

First note that since $Z^{\prime}$ is an open subspace of $Z, X^{\prime}$ is an open subspace of $X$ and $Y^{\prime}$ is an open subspace of $Y$. The condition $A^{\prime} \neq \varnothing$ insures that the $\operatorname{map} f^{\prime}$ is defined in a nontrivial way.

Now let $G^{\prime}$ be an open set of $Z^{\prime}$ in the subspace topology. Then $G^{\prime}=G \cap Z^{\prime}$ where $G$ is open in $Z$; therefore $G^{\prime}$ is open in $Z$. Define $k^{\prime}=k \mid X^{\prime}$. Then $\left(k^{\prime}\right)^{-1}\left(G^{\prime}\right)=k^{-1}\left(G^{\prime}\right)$ is open in $X$ and hence in $X^{\prime}$. Also $G^{\prime} \cap Y^{\prime}$ is open in $Y^{\prime}$, so it follows that $G^{\prime}$ is an open set of $Z^{\prime}$ in the adjunction space topology.

Next, suppose that $G^{\prime}$ is an open set of $Z^{\prime}$ in the adjunction space topology. This means that $\left(k^{\prime}\right)^{-1}\left(G^{\prime}\right)=k^{-1}\left(G^{\prime}\right)$ is open in $X^{\prime}$ (and hence in $X$ ) and that $G^{\prime} \cap Y^{\prime}=G^{\prime} \cap Y$ is open in $Y^{\prime}$ (and hence in $Y$ ). But this simply means that $G^{\prime}$ is open in $Z$, and so $G^{\prime}$ is open in the subspace topology of $Z^{\prime}$.

Thus we have shown that the two topologies coincide, and this completes the proof of the lemma.

The following lemma is well known (see $[6$, p. 15]):

Lemma 2. $Z$ preserves the property $P$ of being (1) Lindelöf (2) normal (3) paracompact (4) countably paracompact normal (5) collectionwise normal.

As a matter of fact, it is known that $Z$ preserves many more properties than these. However, we are interested mainly in these five properties.

The properties listed in Lemma 2 are known to be weakly hereditary, i.e., properties inherited by every closed subspace. It is also well known that the properties (1), (2), (3) and (5) are not hereditary. Examples showing that countable paracompactness is not hereditary seem to be less well known. We shall presently give an example which handles all five cases simultaneously. First, we need to consider a familiar example.

Let $X$ consist of all points $(x, y)$ of the Euclidean plane for which $y \geqq 0$. If $y>0$, define the basis elements at $(x, y)$ to be the open disks with center $(x, y)$ and radius less than $y$. For points $(x, 0)$ on the $x$-axis 
define the basis elements to be the point $(x, 0)$ together with the open disks tangent to the $x$-axis at the point $(x, 0)$. The resulting space $X$ is the classic example of a regular space which is not normal; $X$ is in fact known to be a Tychonoff space. Nonnormality results from the fact that the set $A$ of points on the $x$-axis with rational first coordinate, and the set $B$ of points on the $x$-axis with irrational first coordinate, are disjoint closed subsets of $X$ which cannot be separated by disjoint open sets. Since $X$ is not normal it cannot be paracompact. We shall show that it is not countably paracompact either.

Let $\left\{U_{\lambda}\right\}$ be an open cover of $X$ consisting of basis elements. For each point $(x, 0)$ of $A$ select a member of $\left\{U_{\lambda}\right\}$ containing it; denote the resulting countable collection by $\left\{U_{i}\right\}$. Let $O$ be the union of the remaining members of $\left\{U_{\lambda}\right\}$. Then $\left\{O, U_{i}\right\}$ is a countable open cover of $X$. We claim that $\left\{O, U_{i}\right\}$ has no locally finite open refinement. For suppose that it has a locally finite open refinement $\left\{V_{\mu}\right\}$. Let $\left\{V_{i}\right\}$ be a countable subcollection of $\left\{V_{\mu}\right\}$ containing all the points of $A$ and let $\left\{V_{i}^{\prime}\right\}$ be a collection of basic open neighborhoods of the points of $A$ such that $V_{i}^{\prime} \subset V_{i}$ for each $i$. Now consider any point $(x, 0)$ of $B$. Since $\left\{V_{\mu}\right\}$ is locally finite, there exists a basic open neighborhood $G_{x}$ of $(x, 0)$ which intersects only finitely many of the $V_{i}^{\prime}$ 's. Hence, by taking $G_{x}$ sufficiently small we can assume that $G_{x}$ intersects none of the $V_{i}^{\prime}$ 's. Do this for each point $(x, 0)$ of $B$ and let $G$ be the union of all such $G_{x}$ 's. Let $V^{\prime}$ be the union of all the $V_{i}^{\prime}$ 's. Then $G$ and $V^{\prime}$ are disjoint open sets containing $B$ and $A$ respectively. However, this is a contradiction and shows that the cover $\left\{O, U_{i}\right\}$ can have no locally finite open refinement. Thus we conclude that $X$ is not countably paracompact.

We can now give the example mentioned above.

ExAmple. A space $Y$ which is normal, collectionwise normal, paracompact, countably paracompact, and Lindelöf, but having a subspace with none of these properties.

Let $X$ be the Tychonoff space of the above example and let $Y=\beta(X)$ be the Stone-Cech compactification of $X$. Then $Y$ is compact Hausdorff so it has all the required properties, but its subspace $X$ has none of these properties.

We now continue with the main ideas of the paper.

Lemмa 3. Let $P$ be one of the properties (1)-(5). If every open subset of a space $X$ has property $P$, then every subset of $X$ has property $P$.

Proof. In case $P$ is property (2), property (3), or property (5), the conclusion is known $([1$, p. 268]; $[2$, p. 68]; $[4$, p. 463]). Let us consider the remaining cases. 
Case (1). Lindelöf.

Suppose that every open subset of a space $X$ is Lindelöf, and let $A$ be an arbitrary subset of $X$. Let $\left\{G_{\lambda}^{\prime}\right\}$ be any cover of $A$ by open sets of $A$. Then there exists a collection $\left\{G_{\lambda}\right\}$ of open sets of $X$ such that $G_{\lambda}{ }^{\prime}=G_{\lambda} \cap A$ for each $\lambda$. Thus $G=\bigcup G_{\lambda}$ is an open set of $X$, and as such is a Lindelöf space. Therefore there is a countable subcollection $\left\{G_{i}\right\}$ of $\left\{G_{\lambda}\right\}$ which covers $G$, and if we put $G_{i}^{\prime}=G_{i} \cap A$, then $\left\{G_{i}^{\prime}\right\}$ is a countable subcollection of $\left\{G_{\lambda}{ }^{\prime}\right\}$ which covers $A$. This proves that $X$ is hereditarily Lindelöf.

Case (4). Countably paracompact normal.

Suppose that every open subset of $X$ is countably paracompact normal, and let $A$ be an arbitrary subset of $X$. Then, since we are assuming that every open subset of $X$ is normal, $A$ is normal by case (2). Consider any countable cover $\left\{G_{i}^{\prime}\right\}$ of $A$ by open sets of $A$. Then there exists a countable collection $\left\{G_{i}\right\}$ of open sets of $X$ such that for each $i, G_{i}^{\prime}=G_{i} \cap A$. Define $G=\bigcup_{i=1}^{\infty} G_{i}$. Then $G$ is an open subset of $X$, and as such it is countably paracompact. It follows that $\left\{G_{i}\right\}$ has a locally finite open refinement $\left\{U_{\lambda}\right\}$. Define $U_{\lambda}^{\prime}=U_{\lambda} \cap A$ for each $\lambda$. Then it is clear that $\left\{U_{\lambda}^{\prime}\right\}$ is a locally finite open refinement of $\left\{G_{i}^{\prime}\right\}$. Therefore $A$ is countably paracompact normal, and we have thus shown that $X$ is hereditarily countably paracompact normal.

Let $P$ be a topological property of spaces. If every subspace of a space $X$ has property $P$, we shall say that $X$ has the property Hereditary $P$.

We can now prove, in an efficient manner, the theorem mentioned in the introduction. The proof is obtained by combining Lemmas 1 , 2 and 3.

THEOREM 1. Let $P$ be one of the properties (1)-(5). More generally, let $P$ be any property satisfying the conditions (a) and (b) of the introduction. Then the adjunction space $Z$ preserves the property Hereditary $P$.

Proof. Suppose that the spaces $X$ and $Y$ have the property Heredi$\operatorname{tary} P$, and let $Z$ be the adjunction space obtained by adjoining $X$ to $Y$ by means of a map $f$. Let $Z^{\prime}$ be an open subspace of $Z$.

If $A^{\prime}=A \cap X^{\prime}=\varnothing$, then $X^{\prime} \subset X-A$ and consequently $Z^{\prime}$ is homeomorphic to $X^{\prime}$. Since $X$ has the property Hereditary $P$, it follows that in this case $Z^{\prime}$ has property $P$.

If $A^{\prime} \neq \varnothing$, then by Lemma $1, Z^{\prime}$ is the adjunction space obtained by adjoining $X^{\prime}$ to $Y^{\prime}$ by means of $f^{\prime}$. Both $X^{\prime}$ and $Y^{\prime}$ have property $P$, so by Lemma $2, Z^{\prime}$ has property $P$.

Thus we have shown that every open subset of $Z$ has property $P$. 
Finally, by Lemma $3, Z$ has the property Hereditary $P$. This concludes the proof of our theorem.

According to the example given earlier, Theorem 1 has nontrivial consequences. Note that Iséki's theorem is the special case of Theorem 1 which one gets by taking $P$ to be the property of normality.

The following theorem now follows from Theorem 1 in a standard way $[3$, p. 325$]$ :

Theorem 2. Let the class $Q$ of spaces be one of the following: hereditarily Lindelöf, hereditarily paracompact, hereditarily countably paracompact normal, or hereditarily collectionwise normal. Then

(i) $A Q$-space $Y$ is an ANR $(Q)$ if and only if $Y$ is an NES $(Q)$.

(ii) $A Q$-space $Y$ is an $\mathrm{AR}(Q)$ if and only if $Y$ is an $E S(Q)$.

Let us examine some of these classes of spaces more closely. Hanner has shown [3, p. 333] that a metric ANR (paracompact) is an absolute $G_{\delta}$. By a slight modification of his proof we can prove:

Theorem 3. A metric ANR (hereditarily paracompact) is an absolute $G_{\delta}$.

Proof. Let $Y$ be a metric ANR (hereditarily paracompact). Suppose that $M$ is an arbitrary metric space containing a homeomorphic image of $Y$, which we continue to call $Y$. Define a new space $Z$ as follows: the points of $Z$ are the points of $M$, and a set $U$ in $Z$ is open if and only if $U=O \cup A$, where $O$ is an open set of $M$ and $A \subset Z-Y$. If we can show that $Z$ is hereditarily paracompact, then the theorem will follow as in Hanner's proof. According to Lemma 3, it is sufficient to show that every open subset of $Z$ is paracompact.

Therefore, let $Z^{\prime}$ be any open subset of $Z$, and let $\alpha=\left\{U_{\lambda}\right\}$ be an arbitrary cover of $Z^{\prime}$ by open sets of $Z^{\prime}$. Then each $U_{\lambda}$ is open in $Z$ and as a consequence, $U_{\lambda}=O_{\lambda} \cup A_{\lambda}$, where $O_{\lambda}$ is open in $M$ and $A_{\lambda} \subset Z-Y$. Let $O=\cup O_{\lambda}$. Then $O$, as a subset of the metric space $M$, is paracompact. Therefore there exists a locally finite open refinement $\left\{G_{\mu}\right\}$ of $\left\{O_{\lambda}\right\}$. Complete the collection $\left\{G_{\mu}\right\}$ to an open cover $\beta$ of $Z^{\prime}$ by adding all the points of $Z^{\prime}-O$. These points are all contained in $Z-Y$, so they are open sets of $Z^{\prime}$. Then it is easy to see that $\beta$ is a locally finite open refinement of $\alpha$. This shows that $Z^{\prime}$ is paracompact, and therefore we conclude that $Z$ is hereditarily paracompact. Hence the theorem is proved.

Note that a hereditarily paracompact space is hereditarily collectionwise normal and hereditarily countably paracompact normal. Dowker [1, p. 273] has shown that every hereditarily paracompact space is totally normal. We therefore have the following result: 
COROLlARY 1. Let $Y$ be a metric space which is an ANR (hereditarily collectionwise normal), an ANR (hereditarily countably paracompact normal), or an ANR (totally normal). Then $Y$ is an absolute $G_{\delta}$.

Proof. A metric space is hereditarily paracompact. Hence if the metric space $Y$ is an ANR for one of these classes of spaces, it is an ANR (hereditarily paracompact) and therefore an absolute $G_{\delta}$ by Theorem 3.

That a metric ANR (totally normal) is an absolute $G_{\delta}$ was shown by Mancuso $[8$, p. 17].

Hanner [3, p. 340] has given an example of a space $Y$ which is an ANR (separable metric) but not an absolute $G_{\delta}$. Since $Y$ is a separable ANR (metric) [3, p. 333], it is also an ANR (perfectly normal) [6, p. 87]. But every hereditarily Lindelöf space is perfectly normal, so $Y$ is also an ANR (hereditarily Lindelöf). Hence a metric ANR (hereditarily Lindelöf) need not be an absolute $G_{\delta}$.

Another consequence of Theorem 3 is

CoRollaRy 2. Every metric ANR (hereditarily paracompact) is an ANR (paracompact).

Proof. Let $Y$ be a metric ANR (hereditarily paracompact). Then by Theorem $3, Y$ is an absolute $G_{\delta}$. Since a metric space is hereditarily paracompact, $Y$ is an ANR (metric). But an ANR (metric) which is an absolute $G_{\delta}$ is an ANR (paracompact) [6, p. 87].

\section{REFERENCES}

1. C. H. Dowker, Inductive dimension of completely normal spaces, Quart. J. Math. Oxford (2) 4 (1953), 267-281.

2. J. Dieudonné, Une généralisation des espaces compacts, J. Math. Pures Appl. 23 (1944), 65-76.

3. O. Hanner, Retraction and extension of mappings of metric and non-metric spaces, Ark. Mat. 2 (1952), 315-360.

4. R. E. Hodel, Total normality and the hereditary property, Proc. Amer. Math. Soc. 17 (1966), 462-465.

5. S. T. Hu, Elements of general topology, Holden-Day, San Francisco, Calif., 1964.

6. - Theory of retracts, Wayne State Univ. Press, Detroit, Mich., 1965.

7. K. Iséki, On the hannerization of completely normal spaces, Rev. Fac. Ci. Lisboa Ser. A 3 (1954), 143-146.

8. Vincent Mancuso, Retracts and extension spaces for certain classes of normal spaces, Doctoral Dissertation, Rutgers University, New Brunswick, N. J., 1965.

Western Washington State College 